\title{
Effect of HIP/Ribosomal Protein L29 Deficiency on Mineral Properties of Murine Bones and Teeth
}

\author{
Laura G. Sloofman ${ }^{\mathrm{a}}$, Kostas Verdelis ${ }^{\mathrm{b}}$, Lyudmila Spevak ${ }^{\mathrm{b}}$, Majd Zayzafoon ${ }^{\mathrm{c}}$, Mistuo

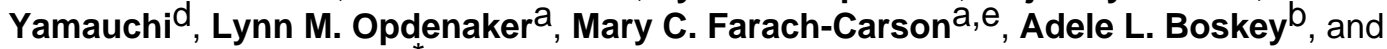 \\ Catherine B. Kirn-Safran ${ }^{\star}, \mathrm{a}$ \\ aDept. Biological Sciences, University of Delaware, Newark, DE 19716, USA \\ bMineralized Tissue Laboratory, Musculoskeletal Integrity Program, Hospital for Special Surgery, \\ New York, NY 10021, USA \\ 'Dept. Pathology, University of Birmingham, Birmingham, AL 35294, USA \\ dNorth Carolina Oral Health Institute, School of Dentistry, University of North Carolina at Chapel \\ Hill, Durham, NC 27709, USA \\ eDept. Biochemistry and Cell Biology, Rice University, Houston, TX 77005, USA
}

\begin{abstract}
Mice lacking HIP/RPL29, a component of the ribosomal machinery, display increased bone fragility. To understand the effect of sub-efficient protein synthetic rates on mineralized tissue quality, we performed dynamic and static histomorphometry and examined the mineral properties of both bones and teeth in HIP/RPL29 knock-out mice using Fourier transform infrared imaging (FTIRI). While loss of HIP/RPL29 consistently reduced total bone size, decreased mineral apposition rates were not significant, indicating that short stature is not primarily due to impaired osteoblast function. Interestingly, our microspectroscopic studies showed that a significant decrease in collagen crosslinking during maturation of HIP/RPL29-null bone precedes an overall enhancement in the relative extent of mineralization of both trabecular and cortical adult bones. This report provides strong genetic evidence that ribosomal insufficiency induces subtle organic matrix deficiencies which elevates calcification. Consistent with the HIP/RPL29-null bone phenotype, HIP/RPL29-deficient teeth also showed reduced geometric properties accompanied with relative increased mineral densities of both dentin and enamel. Increased mineralization associated with enhanced tissue fragility related to imperfection in organic phase microstructure evokes defects seen in matrix protein-related bone and tooth diseases. Thus, HIP/RPL29 mice constitute a new genetic model for studying the contribution of global protein synthesis in the establishment of organic and inorganic phases in mineral tissues.
\end{abstract}

\footnotetext{
(C) 2009 Elsevier Inc. All rights reserved.

*To whom correspondence and proofs should be addressed: Catherine Kirn-Safran, Ph.D., University of Delaware, Dept. Biological Sciences, 310 Wolf Hall, Newark, DE 19716, Tel: 302-831-3249, Fax: 302-831-2281, ckirn@udel.edu .

Publisher's Disclaimer: This is a PDF file of an unedited manuscript that has been accepted for publication. As a service to our customers we are providing this early version of the manuscript. The manuscript will undergo copyediting, typesetting, and review of the resulting proof before it is published in its final citable form. Please note that during the production process errors may be discovered which could affect the content, and all legal disclaimers that apply to the journal pertain.
} 


\section{Keywords}

ribosomal protein knockout; bone mineral content; bone quality; Fourier transform infrared microspectroscopy; teeth

\section{Introduction}

Ribosomal proteins (RPs) play important regulatory functions in the ribosome and help maintain a normal protein synthetic rate. In recent years, several mutations in genes encoding RPs or proteins involved in ribosome biogenesis have been associated with human birth defects with clinical features that include skeletal growth deficiencies and/or deformities [1]. These observations strongly link the fundamental processes of ribosome synthesis, growth, and skeletogenesis. Because of profound effects on embryonic viability, very few RP mutations have been characterized in mammals [2,3]. Our group generated the first viable null mouse mutant model lacking an individual ribosomal protein [4]. In these mutants, the loss of the eukaryote-specific RP of the 60S large ribosomal subunit, HIP/RPL29, resulted in low birth weight accompanied by a global skeletal growth defect that persisted into adulthood. Reduced rates of cellular proliferation and global protein synthesis have been proposed to be the cause of this runting phenotype. Interestingly, the HIP/RPL29-null phenotype in mice is consistent with data reported in yeast (S. cerevisiae), where the deletion of the RPL29 gene is accompanied with delayed growth and protein biosynthesis rates [5]. In this unicellular eukaryote, RPL29 was found to contribute to the proper association between the two ribosomal subunits and, consequently, in mRNA translational efficiency. The absence of an ortholog for RPL29 in prokaryotes supports the idea that RPL29 plays a role in increasing protein translation rates under high demand rather than as a core component of the ribosome translational machinery.

Previously, we reported that HIP/RPL29 expression in the growth plate of developing long bones of mouse embryos is primarily in proliferating and differentiating matrix-producing chondrocytes and is down-regulated in terminally differentiated hypertrophic chondrocytes [6]. Recent comparison of HIP/RPL29-deficient and control growth plates indicated that the reduction in the overall volume occupied by dividing chondrocytes seen in the absence of HIP/ RPL29 is due to the slower progression of chondroprogenitors through the cell cycle [7]. These early changes in the kinetics of osteogenesis are believed to be responsible for the observed bone shortening. Sub-efficient protein synthesis in HIP/RPL29-null bone precursor cells is believed to be responsible for decrease rates of ECM protein production which, in turn, may result in increased fragility in adult HIP/RPL29-deficient long bones [7]. Immunodetection studies demonstrated that HIP/RPL29 is present at the surface of perinuclear membranes of the rough endoplasmic reticulum and also is associated with translationally active ribosomes after polysomal fractionation [4]. HIP/RPL29's predominant subcellular localization at sites where translation of secreted proteins takes place suggests an important role for HIP/RPL29 in modulating the synthesis of secreted proteins including those of the extracellular matrix (ECM).

Bone is a two-phase composite material and its quality is determined by the relative amounts and structure of organic and mineral (hydroxyapatite) phases [8]. During skeletal growth, the rapid but regulated production of ECM protein is crucial for the establishment of normal bone mass and microarchitecture. Dietary or genetic protein deficiencies during skeletal development or in early life can impair bone microstructure and reduce its mechanical properties $[7,9,10]$. Notably, mutations in the genes that encode for the main component of bone ECM, type I collagen, result in a severe increase in fracture risk [11]. In addition, genetic disruption of type I collagen also was found to impact dental health by inducing structural defects in collagen fibrils of dentin [12]. Other mutations in genes encoding for non- 
collagenous proteins such as DMP-1 are also known to affect the relative distribution of organic versus inorganic phases leading to abnormal mineralization in both bone and teeth.

In the present study, we examined the consequences of HIP/RPL29 absence on bone formation potential and analyzed variation in mineral properties of mutant and wild type bones and teeth using Fourier transform infrared microspectrocopic imaging (FTIRI). We hypothesized, based on previous evidence of sub-efficient rates of protein synthesis, that the contribution of the organic phase is reduced in mutant bone and teeth (resulting in a relative increase in mineralto-matrix ratio) and that subtle organic matrix imperfections are responsible for the previously reported fragility phenotype [7].

\section{Materials and methods}

\section{Experimental Animals and Statistics}

Hip/Rpl29 ${ }^{\text {tm2Udel }}$ null and control mice (1, 3, and 6 month-old) in a C57BL6/J background were obtained as described [4]. Because distinct skeletal phenotype differences were not observed between wild type (WT) and heterozygous animals, both genotypes were used as controls for histomorphometric studies [7]. Only WT and null animals were characterized by FTIRI. Statistical comparisons between control and null animals were performed using a twotailed Student's $t$-test. All animal handling experiments complied with the University of Delaware IACUC approved guidelines.

\section{Static and Dynamic Histomorphometry}

After extraction, WT and HIP/RPL29 null mice bones ( $\mathrm{n} \geq 4)$ were fixed immediately for 4-5 days in $4 \%(\mathrm{w} / \mathrm{v})$ phosphate buffered formalin and stored in $70 \%(\mathrm{v} / \mathrm{v})$ ethanol until further processing. For dynamic histomorphometry, bones were double-labeled with calcein at 3 months of age (first label=day 0 , second label=day 8), and euthanized on day 11 post-injection. Histology of the distal metaphysis of femora was performed by the Histomorphometry and Molecular Analysis Core Laboratory of the Center for Metabolic Bone Disease (University of Alabama at Birmingham). Longitudinal sections ( $5 \mu \mathrm{m}$-thick) were cut from MMA plastic embedded blocks along the frontal plane and stained with Goldner's Trichrome stain for static measurements of trabecular indices. Additional $10 \mu \mathrm{m}$-thick sections were obtained and left unstained for dynamic measurements. Histomorphometry was performed using the Bioquant Image Analysis System (R\&M Biometrics, Nashville, TN, USA) as described [13] and as recommended by the Nomenclature Committee of the American Society of Bone and Mineral Research [14]. Single-labeled and double-labeled fluorescent surface measurements were obtained in a cancellous region of bone that measured approximately $2.5 \mathrm{~mm}^{2}$ which was located $0.5-2.5 \mathrm{~mm}$ proximally to the epiphyseal growth cartilage of the femurs and contained only secondary spongiosa.

\section{Bone FTIRI Analysis}

Sections (2-3 $\mu \mathrm{m}$-thick) of all WT and Hip/Rpl2 $9^{\text {tm2Udel }}$ null bone samples were mounted on $\mathrm{BaF} 2$ spectral windows and examined using a FTIRI (Perkin Elmer Spotlight 300 Imaging Spectrometer, Perkin Elmer Instruments, Shelton, CT, USA) with a spectral resolution of 8 $\mathrm{cm}^{-1}$ and a spatial resolution of approximately $7 \mu \mathrm{m}$. Cortical and cancellous bone areas were examined separately, and 3-5 areas of each type were examined per bone. Because no significant difference was observed between genders for most of the bone parameters examined, male and female FTIRI data were combined into either a WT or a RPL29-null category ( $\mathrm{n} \geq 8$ for each group). Background spectra were collected under identical conditions from the same $\mathrm{BaF} 2$ windows. After acquisition, spectra were truncated to allow analysis of the spectral data of interest, baseline was corrected, and the spectral contribution of the polymethyl methacrylate (PMMA)-embedding media was subtracted using ISYS software 
[15]. Spectra were processed to yield images corresponding to infrared band areas, peak height ratios and integrated area ratios by a combination of instrument software and ISYS chemical imaging software (version 3.1, Spectral Dimensions Inc., Olney, MD, USA). In spectral images, all pixels lacking any matrix and/or mineral absorbance were considered devoid of tissue, and set equal to zero and subsequently excluded from all calculations.

The following spectroscopic parameters were determined: mineral-to-matrix ratio [ $v 1, v 3 \mathrm{PO} 4$ band $\left(900-1180 \mathrm{~cm}^{-1}\right) /$ amide I band $\left(1596-1712 \mathrm{~cm}^{-1}\right)$ ], collagen crosslink ratio of intensities $\left(1660 \mathrm{~cm}^{-1} / 1690 \mathrm{~cm}^{-1}\right)$, carbonate-to-mineral ratio [carbonate band $\left(852-900 \mathrm{~cm}^{-1}\right) / \mathrm{v} 1, \mathrm{v} 3$ PO4 band $900-1180 \mathrm{~cm}^{-1}$ ], and crystallinity (ratio of intensities $1030 \mathrm{~cm}^{-1} / 1020 \mathrm{~cm}^{-1}$ ), a value corresponding to the crystallite size and perfection determined by X-ray diffraction line broadening analysis. All spectroscopic results were expressed as histograms, detailing the pixel distribution of the parameters, mean values and standard deviations of the pixel distribution. Corresponding color-coded hyperspectral images (where $\mathrm{x}$, and $\mathrm{y}$ are the locations on the specimen and $\mathrm{z}$ the intensity of the peak or calculated ratio at each pixel) were generated concurrently by ISYS for all parameters.

\section{Tooth Micro-Computed Tomography and FTIRI Analysis}

Right hemimandibles of 3 month-old Hip/Rpl29 $9^{\text {tm2Udel }}$ null and age-matched WT mice ( $\mathrm{n} \geq 11$ for each group) were excised and fixed in $10 \%$ formalin. The hemimandibles were placed in $70 \%$ ethanol and the molar-bearing segment was selected for analysis by micro-computed tomography (micro-CT) on a Scanco $\mu$ CT35 scanner (Scanco Medical, Basserdorf, Switzerland) with a $6 \mu \mathrm{m}$ voxel size. A 0.36 degrees rotation step (360 degrees angular range), $400 \mathrm{~ms}$ exposure and 4 frames per view were used in the scans. The Scanco micro-CT software (HP, DECwindows Motif 1.6) was used for 3D reconstruction and viewing of images.

Anatomical crown volumes of interest were defined with a free-hand tool on sequential slices of the reconstructed molar volumes to include enamel and dentin areas. Root volumes of interest were produced by digital subtraction of the crown volume from the whole molar volume (Fig. 1A). The distribution of mineral densities within the crown and root ROIs was exported as a text file and processed on Excel (Microsoft Corporation, Redmond WA, USA) for crown and root volumes, dentin and enamel volumes, and mean mineral densities of enamel, crown dentin and root dentin. A global pulp-dentin and dentin-enamel mineral density threshold was defined on the distribution of mineral densities within crown volumes of interest for these measurements (Fig. 1B). Because no significant gender difference was observed, male and female micro-CT data were pooled $(\mathrm{n} \geq 11)$ for each genotype examined.

The left hemimandibles of WT and null female animals were partially fixed in $70 \%$ ethanol and were used for the FTIRI analysis of the molar dentin $(n \geq 4)$. The molar-bearing part of the hemimandibles was cut away from the rest of the jaw, dehydrated, embedded in PMMA and $2 \mu \mathrm{m}$-thick sections obtained on BaF2 windows as already described for the bone analyses. Approximately $1 \mathrm{~mm} \times .5 \mathrm{~mm}$ fields around the first and second molar crowns were scanned with a $7 \mu \mathrm{m}$ spatial and $4 \mathrm{~cm}^{-1}$ spectral resolution. Bone and background areas in the scanned fields were masked. The molar crown dentin images were processed for mineral and matrix properties as described above, after a co-addition of all spectra in the masked image process as described before [16]. This co-addition was performed to minimize the effect of thin enamel fragments remaining in the dentin section (enamel essentially is fractured away during sectioning). 


\section{Results}

\section{Morphological properties of HIP/RPL29-/- mice}

Histological analysis of distal metaphyses of 6 month-old HIP/RPL29-null femora showed no obvious morphological abnormalities in their general architecture when compared to control littermates (Fig. 2A-D) other than the overall significant 25\% decrease in total tissue area recently reported [7]. Thus, only a proportionate size decrease and pronounced reduction in bone marrow area are seen in HIP/RPL29-null versus control mice. These observations are consistent with previous comparative micro-CT analyses of HIP/RPL29-deficient bone parameters [7]. Although overall thinning of the growth plate (arrows in Fig. 2 E\&F) and short stature are obvious phenotypic traits of adult null mice, von Kossa staining of HIP/RPL29deficient bone sections did not reveal striking differences in the relative amount of mineral deposit when compared to controls at the macroscopic level (Fig. 2A-D).

To further characterize trabecular bone properties in HIP/RPL29-deficient mice, both static and dynamic histomorphometry analyses were conducted. Because trabeculae are harder to detect in aging adult mice compared with young maturing bone, histomorphometry of cancellous bone was performed in young adult 3 month-old femora (Table 1). Interestingly, even though reduction of global bone geometry is well established in HIP/RPL29-deficient mice, the reduction in the amount of HIP/RPL29-deficient mineralized bone in a given area (BV/TV, $-15.6 \%$ in null vs. controls; $\mathrm{p}>0.05$ ) was not statistically significant. This data was associated with non-significant reduction in trabeculae thickness (Tb. thickness, $-13.9 \%$ in nulls vs. controls; $\mathrm{p}=0.529$ ) relative to controls. Additionally, no difference in the number of osteoblasts/osteoclasts present at the bone surface was noted in the secondary spongiosa between control and HIP/RPL29-null mice (data not shown). Dynamic calcein labeling revealed a significant decrease in the mineralizing surface present in HIP/RPL29 ${ }^{-/-}$bones $(-37.5 \%$; $\mathrm{p}<0.05)$ when compared to controls. However, this decrease did not reach statistical significance after normalization to the bone perimeter present in the corresponding region of interest (MS/BS). Decreases in both the mineral apposition (MAR, $-11.6 \%$ in nulls vs. controls; $\mathrm{p}=0.368$ ) and bone formation (BFR/BS, $-19.2 \%$ in nulls vs. controls; $\mathrm{p}=0.387$ ) rates did not reach statistical significance. Although only subtle impairments were found in the adult bone turnover of null mice, ex vivo investigation performed in bone precursor cells revealed an important decrease in the relative amount of ribosomes present in HIP/RPL29-deficient cells when compared to controls (Supplemental Fig. 1). More importantly, the relative amount of ribosomal RNAs engaged in translational activity, as indicated by their heavier profiles after polysomal fractionation, was significantly decreased in HIP/RPL29 null relative to WT progenitors (Supplemental Fig. 1). Thus, ribosomal insufficiency in HIP/RPL29-null developing bone tissues is believed to induce changes in morphological features and bone formation capacity that, in turn, lead to decreased bone quality in adult mice. To elucidate the factors contributing to the previously described increased brittleness in aging HIP/RPL29 $9^{-1-}$ bones [7], we analyzed the mineral properties of mutant trabecular and cortical bone using vibrational IR microspectroscopy at various ages.

\section{FTIRI of HIP/RPL29-/- and WT bones}

Analysis of the mineral-to-matrix ratio of HIP/RPL29-deficient trabecular bone showed a significant increase in mineral/matrix ratio in 6 month-old mature adult bone when compared to age-matched WT controls [Fig. 3A, $+20.2 \%$ in null $(n=8)$ vs. WT $(n=9) ; p=0.02$ ]. Additionally, a significant $23 \%$ increase in mineral-to-matrix ratio was seen in null trabecular bones from 1 to 6 months $(\mathrm{p}=0.02)$. In contrast, this parameter only significantly increased in WT cortical bone as animals aged [Fig. 3D, $+12.5 \%$ in 6 months $(n=13)$ vs. 1 month $(n=10)$; $\mathrm{p}<0.01]$. In cortices of 6 month-old, the difference between mineral-to-matrix ratios of HIP/ RPL29-deficient $(n=10)$ and control bones $(n=13)$ was smaller, failing to reach statistical 
significance (Fig. 3D, $+2.4 \%$ in nulls vs. WT; $\mathrm{p}=0.489$ ). Interestingly, the mineral-to-matrix ratio of HIP/RPL29-deficient cortical bone was significantly reduced at earlier post-weaning ages when compared to WT controls [Fig. 3D, $-11 \%$ in nulls $(n=10)$ vs. WT $(n=10) ; p=0.025]$. In cortical bone of HIP/RPL29-null animals, the mineral-to-matrix ratio showed a highly significant $30 \%$ increase from 1 to 6 months ( $\mathrm{p}<0.001$ ), whereas WT animals only showed a $12.5 \%$ increase over the same time period $(\mathrm{p}<0.01)$. No genotype dependent differences were found in the mineral-to-matrix ratios at 3 months of age $(n \geq 10)$. To assess whether the relative increase in the mineral phase of adult null bones was also detected at a macroscopic level, we compared the collagen content of null and WT adult femurs using a biochemical approach. No significant change was found in the quantity of collagen (per dry weight of bone) present in HIP/RPL29-deficient bone when compared to the amount present in WT bone (Supplementary Table 1A). Additionally, no genotype-linked difference was noticed in the relative amount of collagen (\% per total protein) and specific amino acid residues (Supplementary Table 1). Similar results were obtained in tibia (data not shown). Interestingly, although the proline/ hydroxyproline ratio was found normal, the lysine to hydroxylysine ratio noticeably increased in HIP/RPL29-deficient bones when compared to WT controls (lys/hyl=2.9 in WT and lys/ hyl=4.4 in null mutants) indicating a shift from hydroxylysine to non-modified lysine residues in the mutant bones (Supplementary Table 1).

In contrast, the XLR parameter indicative of collagen maturity was significantly reduced in HIP/RPL29-deficient bone at 3 months $(n=11)$ in both cortical and trabecular bone areas, when compared with WT $(\mathrm{n}=10)$ by approximately $10 \%$ and $11 \%$, respectively (Fig. $4 ; \mathrm{p}<0.05)$. This decrease in the XLR parameter at 3 month of age was slightly more pronounced in null female trabecular bone than in equivalent null male bone and was diminished by nearly $15 \%$ when compared with age-matched WT female littermates ( $\mathrm{p}<0.05$, data not shown). Interestingly, such a decrease in the XLR parameter was also more apparent in 3 month-old female growth plates (data not shown). By 6 months, the XLR decrease seen in maturing 3 month-old null bones was overcome (Fig. 3A, D) and the overall increase in the XLR parameter for null bones was statistically significant $(+17 \%, 1$ month vs. 6 months) and more pronounced than for control bones.

The carbonate-to-mineral ratio which increases with age and is related to mineral solubility was slightly elevated in cortices and trabeculae of young HIP/RPL29 null animals but remained equivalent to the carbonate-to-mineral ratio of WT controls at older ages ( $\mathrm{n} \geq 10$, data not shown). No significant changes were noted in the size and perfection of hydroxyapatite crystals in any mutant bone tissue examined as indicated by relatively small changes in the crystallinity parameter over time and when compared with WT controls ( $\mathrm{n} \geq 10$, data not shown). With the exception of the XLR parameter in HIP/RPL29 ${ }^{-/-}$trabecular bone where the decrease in

females was more prominent than in males, no significant gender-dependent differences were noted in null bones when compared to WT controls for all the four parameters examined.

\section{Micro-CT of wild type and HIP/RPL29-/- teeth}

Comparative analysis of the structural properties of 3 month-old HIP/RPL29-null $(n=12)$ and WT $(\mathrm{n}=10)$ first molars by micro-CT showed significant decrease of root volume $(-11.3 \%$, $\mathrm{p} \leq 0.001$, Table 2). A similar observation was made in the second molar where the volume of HIP/RPL29-null roots also was significantly decreased compared to age-matched WT controls (Table 2B, $-5.3 \%$ in nulls vs. WT; $\mathrm{p}<0.05$ ). Although the dentin and enamel volumes were not changed between genotypes, small but significant increases in mineral density were found in HIP/RPL29-deficient first molars relative to their WT counterparts (Table $2 \mathrm{~A},+2.1 \%$ for dentin and $+1.6 \%$ for enamel; $\mathrm{p} \leq 0.001$ ). A significant increase in dentin mineral content also was found in second molars (Table $2 \mathrm{~B},+1.8 \%$ in nulls vs. WT; $\mathrm{p}<0.01$ ). Although no measurement could be obtained for cementum, a collagenous-based component of tooth roots, 
hypercementosis was found in two of the HIP/RPL29-deficient second molar examined (one male and one female, $n=12$ ) but was never observed in any of the wild type control second molar (Fig. 5).

In first molars, no significant gender-dependent differences were noted in HIP/RPL29deficient when compared with WT controls for any of the parameters examined. Although trends were conserved in WT and HIP/RPL29-null second molars of both genders, differences relative to gender-matched WT were more pronounced in null females than in null males (data not shown). For this reason, further analysis of tooth molecular composition by Fourier transform microspectroscopy was performed only on female teeth.

\section{FTIRI of wild type and HIP/RPL29-/- teeth}

In agreement with the micro-CT results for dentin mineral density, the average mineral-tomatrix ratio in dentin increased in first and second molars by $6 \%$ and $4 \%$, respectively $(n=5)$ when compared to corresponding WT molars $(n=5)$; however these differences were not statistically significant (Table 3 ).

On the other hand, crystallinity was significantly reduced in HIP/RPL $29^{-1-}$ first molars (-8\% in null vs. WT; $\mathrm{p}<0.05)$ but not changed in HIP/RPL2 $9^{-/-}$second molars. There was no significant difference among genotypes for the other FTIRI parameters in either first or second molars.

\section{Discussion}

Bones and teeth are mineralized tissues composed of an organic matrix secreted by specialized cells; hydroxyapatite crystals are embedded in that matrix in an organized fashion. Coordinated synthesis and secretion of type I collagen and non-collagenous proteins are essential for establishment of normal tissue mass and mineral distribution in bone, dentin, and root cementum [17-20]. Thus, it is not surprising that disruption of specific components of the protein synthetic machinery during development has dramatic consequences on growth and quality of the adult bone tissue $[2,7,21]$. In this study, we show that sub-optimal protein biosynthesis rates [4] in HIP/RPL29-null mice resulted in a proportionate short stature phenotype that is not associated with a significant decrease in bone apposition rates and collagen quantity. Additionally, although residual growth plates of HIP/RPL29-deficient adult bones appeared thinner than in WT controls, columnar chondrocytes and the chondro-osteo junction remained normally arranged. Thus, the reported $25 \%$ reduction in bone volume of HIP/RPL29-deficient mice is not a direct consequence of changes in osteoblast function and bone remodeling but may rather be due to reduced ECM protein production and delayed osteoprogenitor differentiation during bone development [7]. This observation is consistent with reduced in vitro differentiation potential and decreased translational efficiency in HIP/ RPL29-null osteoblast precursors, and with short stature phenotypes found in DiamondBlackfan patients carrying other $R P$ gene mutations known to affect differentiation of bone marrow precursors $[7,22,23]$.

Our recent work indicated that elevated mineral density in HIP/RPL29-deficient cortices likely is responsible for increased fragility of long bone shafts [7]. This discovery is in agreement with the idea that variation in bone growth patterns can be linked with alterations in biomechanical properties [24]. Here we analyzed further the causes of diminished bone quality in HIP/RPL29-deficient mice by defining the relative distribution of organic versus inorganic phases and reporting important changes in the matrix composition of both cortical and trabecular bone using FTIRI. This study shows that homozygous deletion of the Hip/Rpl29 gene in mice is accompanied by an increase in the relative mineral content of adult bone compared to their age matched WT counterparts. This increase in mineral-to-matrix ratio is 
reminiscent of local microhardness and increased mineral content noted in a murine model (oim) of osteogenesis imperfecta (OI) in which the gene encoding for the major matrix component, alpha 2 type I collagen gene, is disrupted resulting a higher mineral-to-matrix ratio $[11,25,26]$. However, unlike in oim/oim mice in which specific disorganization of collagen matrix is observed, HIP/RPL29 null mutation does not induce changes in terms of global collagen content and composition in bone. Thus, the observed imbalance in the ratio of the two bone phases and poor mechanical properties of HIP/RPL29-deficient bones are more likely attributable to more subtle changes in organic matrix arrangement. Typically, stabilization of bone tissue by elevation of its mineral content comes at the cost of increased brittleness and raises fracture risk. Likewise, sub-efficient protein biosynthesis in HIP/RPL29-null developing bone tissues results in abnormal mineral-to-matrix ratio and increased mineral content that may contribute to increased fragility [7].

Although the contribution of the mineral phase is believed to be the primary modulator of bone's toughness, this study shows strong genetic evidence that the organic phase is also an important determinant of bone's unique material properties and is in agreement with studies performed by others [25,27]. Here, we demonstrate that the bone matrix of a mouse model of ribosomal insufficiency displays collagen crosslinking imperfections during the maturation phase followed by a hypermineralization phenotype at an age when peak bone mass is reached. Similar observations were made in the primary spongiosa of developing growth plate cartilage that serves as a template for endochondral bone formation. Since bone collagen crosslinks utilize hydroxyproline for stability, a decrease in the relative level of hydroxylysine versus lysine supports the idea that HIP/RPL29 mutant bone quality is affected. Because collagen forms the template upon which the mineral is deposited and collagen fibrils within the HIP/ RPL29 knockout are presumably less compacted (lower XLR/less mature), local increase in sites available for mineral deposition could occur [18]. However, additional molecular evaluations will be needed to determine whether disarrangement of the collagen network during bone maturation is responsible for subsequent matrix-to-mineral ratio decrease in fully mature bone. Future studies will consist of determining if imperfections in the microstructure of the organic phase are related to abnormal mineralization foci using electron microscopy during critical stages of bone maturation. Interestingly, although the XLR parameter was diminished in both male and female mice, females' trabecular bone displayed even lower XLR values indicating that sex partially modulated the effect of the genotype for this parameter. This data is in accordance with previous observations that HIP/RPL29 null males can partially compensate for decreased geometric properties but not null females [7].

Thus, global ribosomal insufficiency during bone growth appears to be at the origin of abnormal mineral deposition at older age suggesting an indirect role for HIP/RPL29 in the regulation of matrix mineralization. In contrast, specific non-collagenous proteins, such as osteopontin and DMP-1 were found to have a more active role to play in biomineralization as either inhibitor or initiator/regulator of mineral formation and crystal growth, respectively [28-30]. This observation agrees with our finding that the crystallinity parameter is unchanged in HIP/ RPL29-deficient bone when compared to age-matched controls at all stages examined.

To determine the effect of HIP/RPL29 depletion on another mineralized tissue, we compared the structural and compositional properties of young adult teeth and noticed that the phenotype in the HIP/RPL29-null tooth is quite similar to that of the bone. First, the decrease in microCT measurements for HIP/RPL29 $9^{-/}$first and second molars versus age-matched WT teeth is in good agreement with the global skeletal growth deficiencies reported previously [4].

Interestingly, this geometrical decrease was more pronounced in the root areas, and might be due to the fact that root morphogenesis takes place after crown formation postnatally and cannot be as easily rescued through maternal circulation and lactation. Second, the club shape hypercementosis phenotype found in two out of the twelve HIP/RPL29-null first molar roots 
examined is indicative of an increase in cementum deposition and the presence of abnormal mineralization foci [31]; which is consistent with the hypermineralization phenotype found in HIP/RPL29-deficient bones. This idea is reinforced by a significant increase in the mineral density of dentin, and, to a lesser extent of enamel, in the first molars of HIP/RPL29-null mice when compared to age-matched controls. In the second molar, which is developmentally delayed relative to the first molar, only dentin mineral density was significantly increased in mutant teeth versus WT. Like for bone, this data indicates an elevated risk of tooth microfracture and fragility associated with aging in mice with altered protein synthesis rates. Similarly, increased hardness and dentin fragility occurs in mice which exhibit mutations in either the major structural protein present in dentin (type I collagen) or non collagenous protein such as DMP-1 [28,32]. The lower genotype effect observed in HIP/RPL29-deficient enamel might be inherent to the nature of enamel which contains little protein, none of which is collagen [33].

The detailed mineral analysis of the teeth based on infrared microspectroscopy showed that the mineral-to-matrix ratio was noticeably increased in HIP/RPL29 deficient teeth whereas the crystallinity, which is an indicator of crystal size and purity was significantly decreased in HIP/ RPL29-null first molars relative to age-matched WT controls. Smaller crystal size in mutant teeth is suggestive of a developmental delay when compared with controls. Although an increase in mineral density with decreased crystal size has not been reported previously in teeth it has been described in cases of osteopetrotic bone diseases [34]. Additional studies will be necessary to determine whether the reduction in crystallinity in HIP/RPL29 null mutant teeth is corrected during aging and results primarily from delayed morphogenesis.

In summary, we determined that deficiency in a component of the protein synthesis machinery induces defects in the relative distribution and/or structural organization of the mineral and organic phases of bone tissue, dentin and possibly cementum that subsequently lead to the relative hypermineralization of both bones and teeth, respectively. Elevated material properties and increase in fragility of mineralized tissues evokes defects reminiscent of matrix proteinrelated genetic bone and tooth diseases making this model useful for studying the effects of protein deficiencies on mineralization.

\section{Supplementary Material}

Refer to Web version on PubMed Central for supplementary material.

\section{Acknowledgments}

The authors wish to acknowledge Ms. Julie Mis at the OLAM for her assistance with animal husbandry and Mr. Daniel Oristian for imaging mature bone sections. We also thank the University of Alabama at Birmingham, Center for Metabolic Bone Disease, Histomorphometry and Molecular Analysis Core Laboratory, NIH Grant P30-AR46031, for help with the histology and histomorphometry. This work was supported by NIH P20-RR016458 (to C.B. Kirn-Safran and M.C. Farach-Carson), NIH P30-AR046121 (to A.L. Boskey), NIH 1S10RR02547 (to HSS Imaging Core), and NIH R01-AR053898 (to M. Zayzafoon).

\section{References}

1. Liu JM, Ellis SR. Ribosomes and marrow failure: coincidental association or molecular paradigm? Blood 2006;107:4583-4588. [PubMed: 16507776]

2. Oliver ER, Saunders TL, Tarle SA, Glaser T. Ribosomal protein L24 defect in belly spot and tail (Bst), a mouse Minute. Development 2004;131:3907-3920. [PubMed: 15289434]

3. Panic L, Tamarut S, Sticker-Jantscheff M, Barkic M, Solter D, Uzelac M, Grabusic K, Volarevic S. Ribosomal protein S6 gene haploinsufficiency is associated with activation of a p53-dependent checkpoint during gastrulation. Mol Cell Biol 2006;26:8880-8891. [PubMed: 17000767] 
4. Kirn-Safran CB, Oristian DS, Focht RJ, Parker SG, Vivian JL, Carson DD. Global growth deficiencies in mice lacking the ribosomal protein HIP/RPL29. Dev Dyn 2007;236:447-460. [PubMed: 17195189]

5. DeLabre ML, Kessl J, Karamanou S, Trumpower BL. RPL29 codes for a non-essential protein of the 60S ribosomal subunit in Saccharomyces cerevisiae and exhibits synthetic lethality with mutations in genes for proteins required for subunit coupling. Biochim Biophys Acta 2002;1574:255-261. [PubMed: 11997090]

6. Miller SA, Brown AJ, Farach-Carson MC, Kirn-Safran CB. HIP/RPL29 down-regulation accompanies terminal chondrocyte differentiation. Differentiation 2003;71:322-336. [PubMed: 12919102]

7. Oristian DS, Sloofman LG, Zhou X, Wang L, Farach-Carson MC, Kirn-Safran CB. Ribosomal protein L29/HIP deficiency delays osteogenesis and increases fragility of adult bone in mice. J Orthop Res 2009;27:28-35. [PubMed: 18661500]

8. Burr DB. The contribution of the organic matrix to bone's material properties. Bone 2002;31:8-11. [PubMed: 12110405]

9. Alippi RM, Meta MD, Bozzini C, Olivera MI, Ferretti JL, Bozzini CE. Dynamics of recovery of morphometrical variables and pQCT-derived cortical bone properties after a short-term protein restriction in maturing rats. Growth Dev Aging 2002;65:67-72. [PubMed: 11936277]

10. Jepsen KJ, Pennington DE, Lee YL, Warman M, Nadeau J. Bone brittleness varies with genetic background in A/J and C57BL/6J inbred mice. J Bone Miner Res 2001;16:1854-1862. [PubMed: 11585350]

11. Grabner B, Landis WJ, Roschger P, Rinnerthaler S, Peterlik H, Klaushofer K, Fratzl P. Age- and genotype-dependence of bone material properties in the osteogenesis imperfecta murine model (oim). Bone 2001;29:453-457. [PubMed: 11704498]

12. Kim JW, Simmer JP. Hereditary dentin defects. J Dent Res 2007;86:392-399. [PubMed: 17452557]

13. O'Connor RD, Zayzafoon M, Farach-Carson MC, Schanen NC. Mecp2 deficiency decreases bone formation and reduces bone volume in a rodent model of Rett syndrome. Bone 2009;45:346-356. [PubMed: 19414073]

14. Parfitt AM, Drezner MK, Glorieux FH, Kanis JA, Malluche H, Meunier PJ, Ott SM, Recker RR. Bone histomorphometry: standardization of nomenclature, symbols, and units. Report of the ASBMR Histomorphometry Nomenclature Committee. J Bone Miner Res 1987;2:595-610. [PubMed: 3455637]

15. Gourion-Arsiquaud S, West PA, Boskey AL. Fourier transform-infrared microspectroscopy and microscopic imaging. Methods Mol Biol 2008;455:293-303. [PubMed: 18463826]

16. Verdelis K, Ling Y, Sreenath T, Haruyama N, MacDougall M, van der Meulen MC, Lukashova L, Spevak L, Kulkarni AB, Boskey AL. DSPP effects on in vivo bone mineralization. Bone 2008;43:983-990. [PubMed: 18789408]

17. Kocher MS, Shapiro F. Osteogenesis imperfecta. J Am Acad Orthop Surg 1998;6:225-236. [PubMed: 9682085]

18. Boskey AL, Young MF, Kilts T, Verdelis K. Variation in mineral properties in normal and mutant bones and teeth. Cells Tissues Organs 2005;181:144-153. [PubMed: 16612080]

19. MacDougall M, Dong J, Acevedo AC. Molecular basis of human dentin diseases. Am J Med Genet A 2006;140:2536-2546. [PubMed: 16955410]

20. Zhang X, Zhao J, Li C, Gao S, Qiu C, Liu P, Wu G, Qiang B, Lo WH, Shen Y. DSPP mutation in dentinogenesis imperfecta Shields type II. Nat Genet 2001;27:151-152. [PubMed: 11175779]

21. Wei J, Sheng X, Feng D, McGrath B, Cavener DR. PERK is essential for neonatal skeletal development to regulate osteoblast proliferation and differentiation. J Cell Physiol 2008;217:693707. [PubMed: 18683826]

22. Pederson T. Ribosomal protein mutations in Diamond-Blackfan anemia: might they operate upstream from protein synthesis? Faseb J 2007;21:3442-3445. [PubMed: 17586729]

23. Gazda HT, Kho AT, Sanoudou D, Zaucha JM, Kohane IS, Sieff CA, Beggs AH. Defective ribosomal protein gene expression alters transcription, translation, apoptosis, and oncogenic pathways in Diamond-Blackfan anemia. Stem Cells 2006;24:2034-2044. [PubMed: 16741228]

24. Price C, Herman BC, Lufkin T, Goldman HM, Jepsen KJ. Genetic variation in bone growth patterns defines adult mouse bone fragility. J Bone Miner Res 2005;20:1983-1991. [PubMed: 16234972] 
25. Miller E, Delos D, Baldini T, Wright TM, Pleshko Camacho N. Abnormal mineral-matrix interactions are a significant contributor to fragility in oim/oim bone. Calcif Tissue Int 2007;81:206-214. [PubMed: 17660935]

26. Camacho NP, Landis WJ, Boskey AL. Mineral changes in a mouse model of osteogenesis imperfecta detected by Fourier transform infrared microscopy. Connect Tissue Res 1996;35:259-265. [PubMed: 9084664]

27. Burstein AH, Zika JM, Heiple KG, Klein L. Contribution of collagen and mineral to the elastic-plastic properties of bone. J Bone Joint Surg Am 1975;57:956-961. [PubMed: 1184645]

28. Ling Y, Rios HF, Myers ER, Lu Y, Feng JQ, Boskey AL. DMP1 depletion decreases bone mineralization in vivo: an FTIR imaging analysis. J Bone Miner Res 2005;20:2169-2177. [PubMed: 16294270]

29. Boskey AL, Spevak L, Paschalis E, Doty SB, McKee MD. Osteopontin deficiency increases mineral content and mineral crystallinity in mouse bone. Calcif Tissue Int 2002;71:145-154. [PubMed: 12073157]

30. Tartaix PH, Doulaverakis M, George A, Fisher LW, Butler WT, Qin C, Salih E, Tan M, Fujimoto Y, Spevak L, Boskey AL. In vitro effects of dentin matrix protein-1 on hydroxyapatite formation provide insights into in vivo functions. J Biol Chem 2004;279:18115-18120. [PubMed: 14769788]

31. Pinheiro BC, Pinheiro TN, Capelozza AL, Consolaro A. A scanning electron microscopic study of hypercementosis. J Appl Oral Sci 2008;16:380-384. [PubMed: 19082395]

32. Lopez Franco GE, Huang A, Pleshko Camacho N, Stone DS, Blank RD. Increased Young's modulus and hardness of Col1a2oim dentin. J Dent Res 2006;85:1032-1036. [PubMed: 17062745]

33. Bonar LC, Shimizu M, Roberts JE, Griffin RG, Glimcher MJ. Structural and composition studies on the mineral of newly formed dental enamel: a chemical, $\mathrm{x}$-ray diffraction, and 31P and proton nuclear magnetic resonance study. J Bone Miner Res 1991;6:1167-1176. [PubMed: 1666806]

34. Boskey AL. Assessment of bone mineral and matrix using backscatter electron imaging and FTIR imaging. Curr Osteoporos Rep 2006;4:71-75. [PubMed: 16822406] 

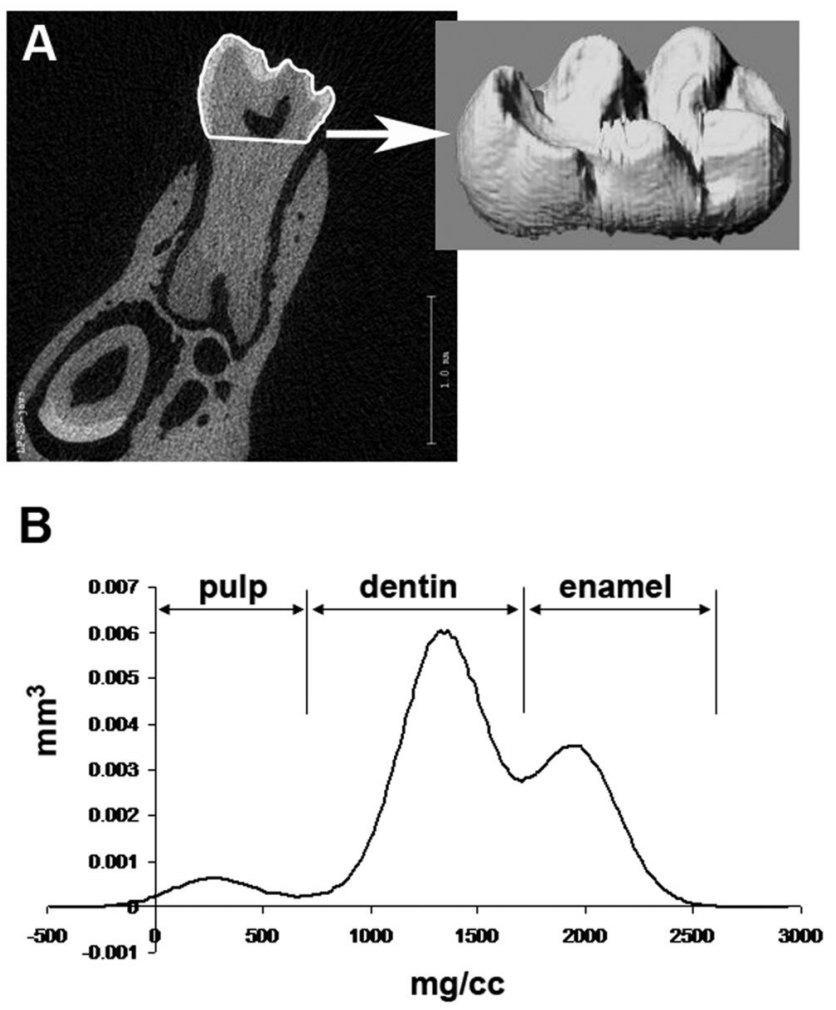

Figure 1. Micro-CT of molars

A: Definition of the crown volume of interest on sequential slices-3D rendering of the crown volume (right) B: Distribution of mineral densities within a crown volume. 

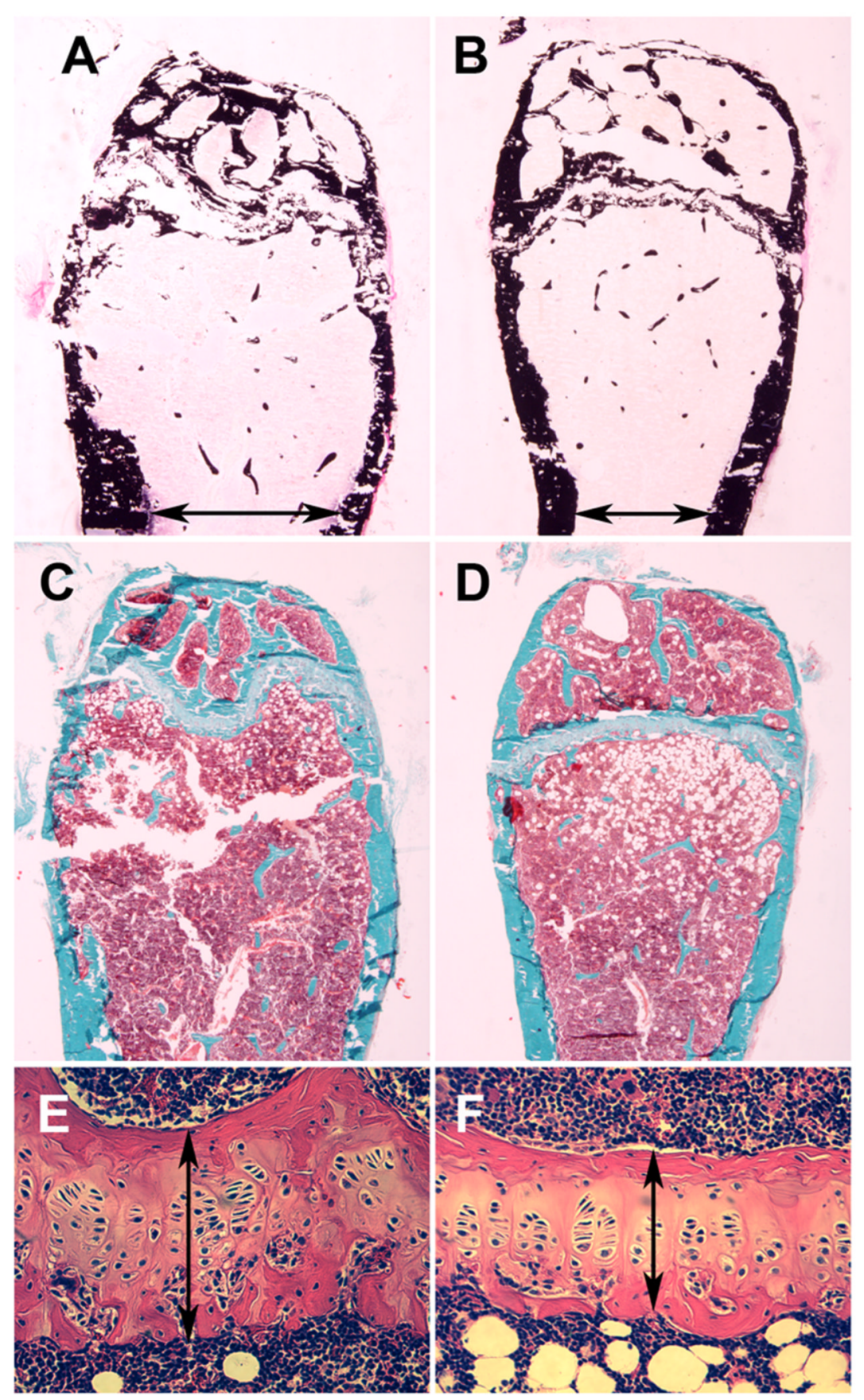

Figure 2. Histological comparison of mature bone in HIP/RPL29-deficient and control mice Consecutive non-decalcified von Kossa (A, B) and Goldner's trichrome (C, D) -stained sections of the epiphyseal bone, growth plate, and metaphyseal bone of 6-month old HIP/ $R P L 29^{-/-}(\mathrm{B}, \mathrm{D} ; \mathrm{n}=6)$ and controls $(\mathrm{A}, \mathrm{C} ; \mathrm{n}=4)$ mice. The decrease of mineral deposits in the absence of HIP/RPL29 reflected a decrease in the global geometric properties of null mutant bone relative to controls. Note the rapid narrowing of the bone marrow cavity (horizontal arrows) in null mutants (B, D). Closer examination of HIP/RPL29-1- tibial growth plates (E, F) after decalcification and hematoxylin/eosin staining indicated well organized columnar arrangement of chondrocytes and reduced overall thickness compared to controls (vertical arrows in $\mathrm{E}$ and $\mathrm{F}$ ). 

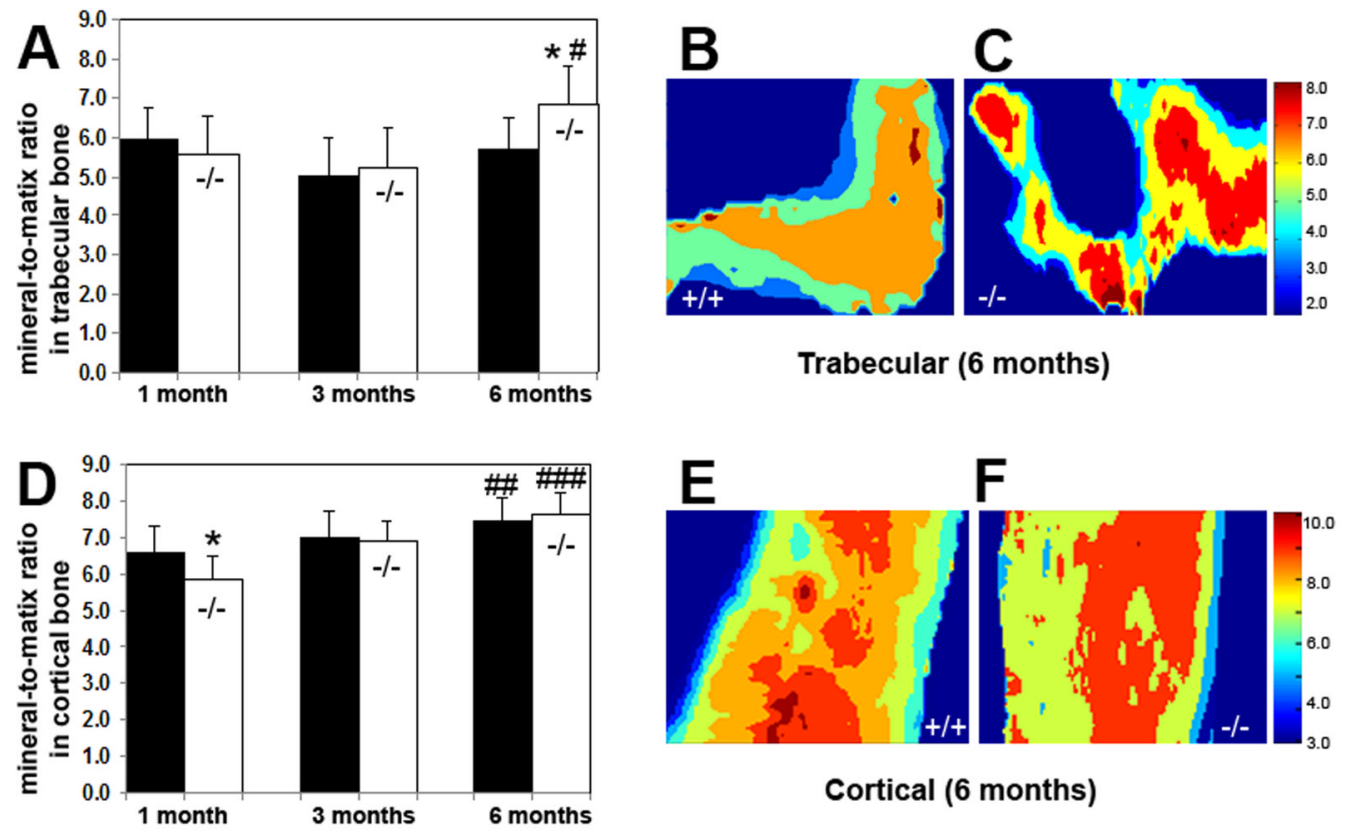

Figure 3. FTIR microspectroscopic analysis of the bone mineral-to-matrix ratio of HIP/RPL29null and age-matched WT mice

Mean values obtained from at least eight animals for the area of phosphate band and the area of amide I band at sites below the growth plate and at mid-shaft for trabecular and cortical bone, respectively (A, D). Whereas the mineral-to-matrix ratio $\left(v 1, v 3 \mathrm{PO}_{4}\right.$ area /amide I area) is first below average in HIP/RPL29-deficient younger bone when compared with WT it becomes increased in older bone, with statistical significance in 6 month-old trabecular bones $\left(+20.2 \%\right.$ in nulls, $n=8$ vs. WT, $n=9$; $\left.*^{p}<0.05\right)$. Typical spectral infrared images of 6 monthold trabecular $(\mathrm{B}, \mathrm{C})$ and cortical bone $(\mathrm{E}, \mathrm{F})$ are shown. For mean value comparison in the same genotype in 6 months $(n \geq 8)$ versus 1 month $(n \geq 10)$, the $p$ values are as follows: $\# p<0.05$, \#\# p<0.01, \#\#\# p<0.001. 

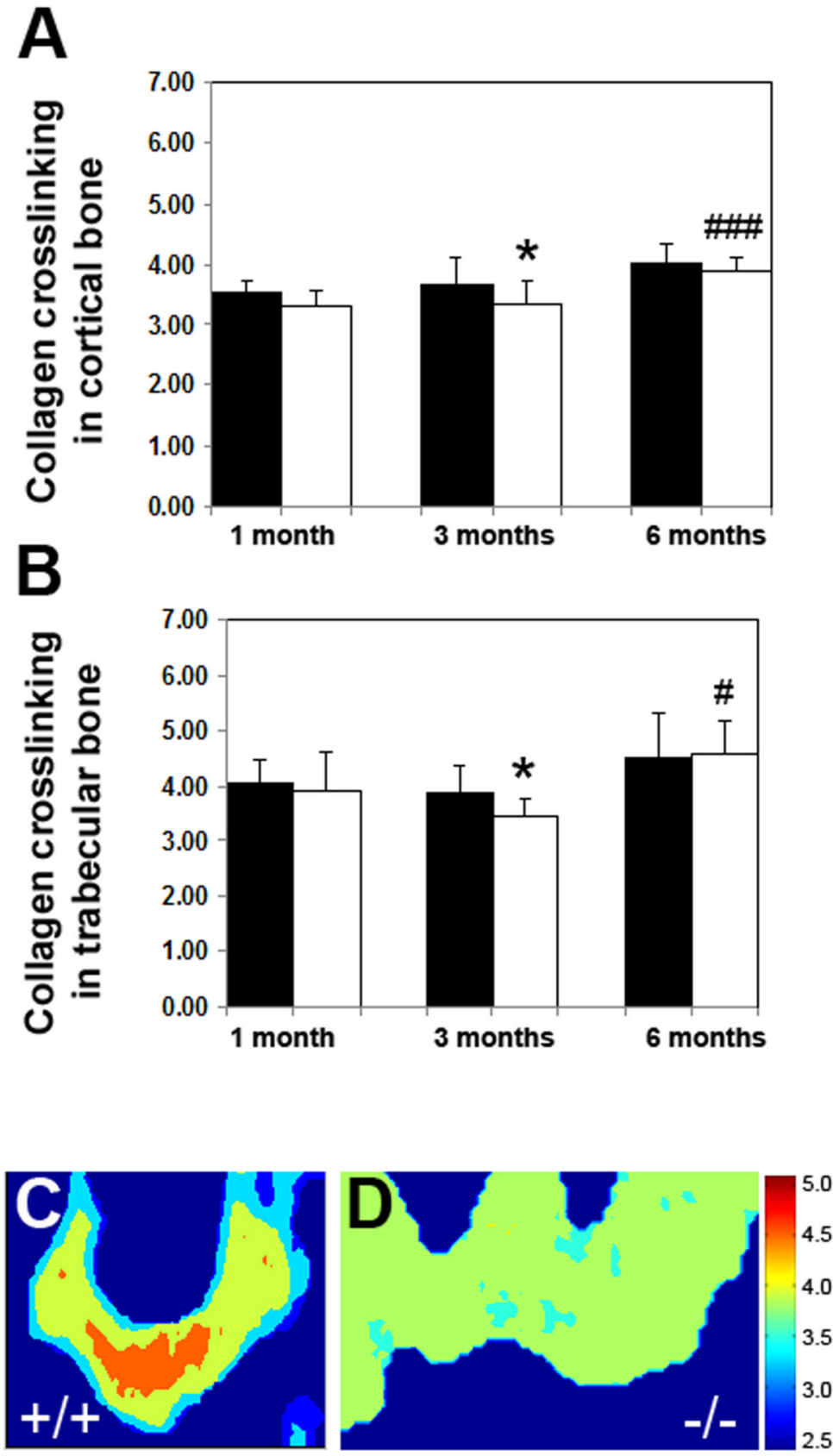

Trabecular (3 months)

Figure 4. FTIR microspectroscopic analysis of the collagen crosslinking parameter in HIP/RPL29null and age-matched WT bones

The mean values obtained from at least eight animals in cortical (A) and trabecular bone (B) for collagen crosslinking (intensity ratio 1660/1690) are significantly reduced in HIP/RPL29deficient bone $(n=11)$ at 3 months $(* \mathrm{p}<0.05)$ compared with WT $(n=10)$ but restored to control levels in older bones. Significant increase in collagen crosslinking is seen in older null animals compared to nulls at post-weaning age (\# $\mathrm{p}<0.05$ and \#\#\# $\mathrm{p}<0.001$ in 6 months vs. 1 month, $n=10)$. Typical spectral infrared images of 3 month-old trabecular $(C, D)$ are shown. Highest values normally present in the center of the trabeculae in WT are absent in HIP/RPL29-deficient bones. 


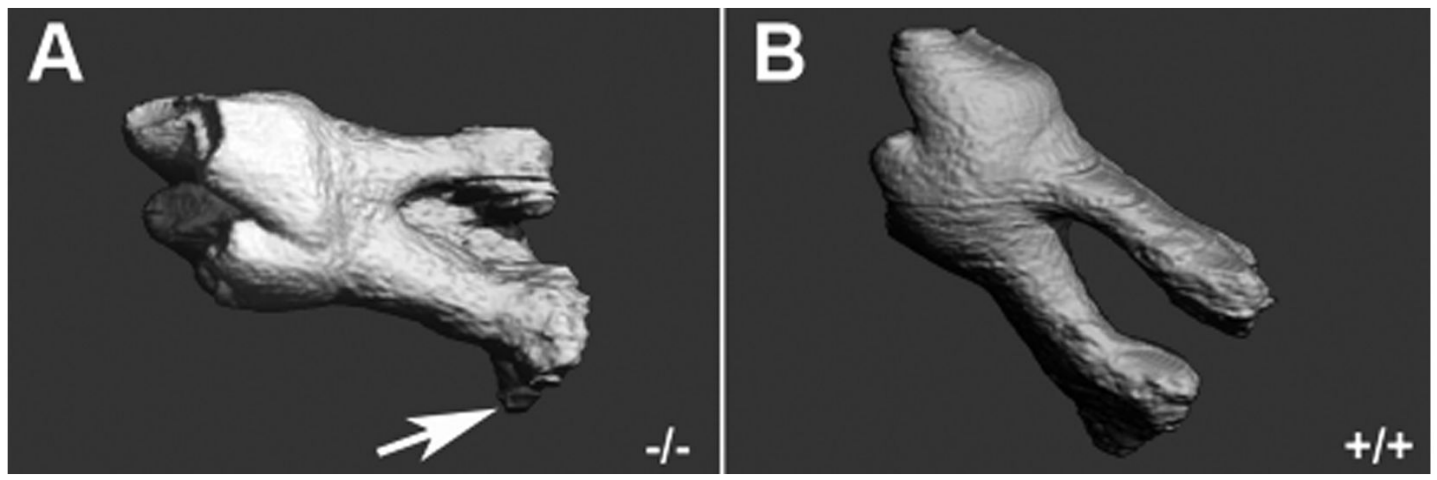

Figure 5. Hypercementosis in HIP/RPL29-null second molars

Tridimensional micro-CT reconstruction of HIP/RPL29 ${ }^{-1-}(\mathrm{A}, \mathrm{n}=12)$ and WT $(\mathrm{B}, \mathrm{n}=10)$ second molars at three months. The arrow indicates a club shape hypercementosis phenotype in a HIP/RPL29-deficient molar. 
Table 1

Static and Dynamic Histomorphometry

\begin{tabular}{ccc}
\hline & Controls & Hip/Rpl29 $^{-/-}$ \\
\hline BV/TV & $10.28(+/-6.88)$ & $8.67(+/-3.86)$ \\
Bone Perimeter (BS) mm & $6.01(+/-4.53)$ & $6.03(+/-1.45)$ \\
BS/BV (mm-1) & $62.96(+/-10.48)$ & $69.33(+/-14.96)$ \\
BS/TV (mm-1) & $5.57(+/-2.45)$ & $5.62(+/-1.69)$ \\
Trabecular Thickness $(\mu \mathrm{m})$ & $34.87(+/-12.49)$ & $30.01(+/-7.04)$ \\
Trabecular Number $(\mathrm{mm}-1)$ & $2.78(+/-1.23)$ & $2.81(+/-0.85)$ \\
Trabecular Space $(\mu \mathrm{m})$ & $385.69(+/-191.54)$ & $360.70(+/-158.94)$ \\
Mineralizing surface/MS $(\mathrm{mm})$ & $3.65(+/-1.13)$ & $\mathbf{2 . 2 8}(+/-\mathbf{1 . 2 2})$ \\
MS/BS & $35.56(+/-9.33)$ & $33.03(+/-13.10)$ \\
Mineral Apposition Rate $(\mathrm{MAR}) \mu \mathrm{m} /$ day & $1.54(+/-0.29)$ & $1.36(+/-0.43)$ \\
Bone Form Rate (BFR/BS) $\mu \mathrm{m} /$ day & $0.55(+/-0.22)$ & $0.45(+/-0.21)$ \\
\hline
\end{tabular}

${ }^{*} \mathrm{p}<0.05$; Controls consist of both WT and HIP/RPL29 ${ }^{+/-}$mice. Hip/Rpl29 $9^{-/-}$and controls $(\mathrm{n} \geq 4)$. Data are mean $+/-$ SD. 
Table 2

\section{Comparative tooth micro-computed tomography analysis}

\begin{tabular}{|c|c|c|c|}
\hline & Controls (WT) & HIP/RPL 29-1- & $\begin{array}{c}\% \text { change vs. } \\
\text { control }\end{array}$ \\
\hline \multicolumn{4}{|l|}{ A. First Molar } \\
\hline Total volume (mm3) & $1.18(+/-0.05)$ & $1.09(+/-0.07)$ & $-7.6 \% * *$ \\
\hline Crown volume (mm3) & $0.47(+/-0.02)$ & $0.47(+/-0.04)$ & No change \\
\hline Root volume (mm3) & $0.71(+/-0.04)$ & $0.63(+/-0.06)$ & $-11.3 \% * * *$ \\
\hline Dentin volume (mm3) & $0.32(+/-0.01)$ & $0.31(+/-0.03)$ & No change \\
\hline Enamel volume (mm3) & $0.12(+/-0.01)$ & $0.12(+/-0.01)$ & No change \\
\hline Dentin mineral density (mg/cc) & $1268(+/-15)$ & $1295(+/-17)$ & $2.1 \% * * *$ \\
\hline Enamel mineral density (mg/cc) & $1910(+/-26)$ & $1940(+/-27)$ & $1.6 \% * *$ \\
\hline \multicolumn{4}{|l|}{ B. Second Molar } \\
\hline Total volume $(\mathrm{mm} 3)$ & $0.66(+/-0.04)$ & $0.64(+/-0.03)$ & $-3 \%$ \\
\hline Crown volume (mm3) & $0.28(+/-0.01)$ & $0.28(+/-0.02)$ & No change \\
\hline Root volume (mm3) & $0.38(+/-0.03)$ & $0.36(+/-0.02)$ & $-5.3 \% *$ \\
\hline Dentin volume (mm3) & $0.17(+/-0.01)$ & $0.17(+/-0.01)$ & No change \\
\hline Enamel volume (mm3) & $0.08(+/-0.01)$ & $0.09(+/-0.01)$ & $12.5 \%$ \\
\hline Dentin mineral density (mg/cc) & $1290(+/-24)$ & $1313(+/-14)$ & $1.8 \% * *$ \\
\hline Enamel mineral density (mg/cc) & $1934(+/-43)$ & $1955(+/-24)$ & $1.1 \%$ \\
\hline
\end{tabular}

Hip/Rpl29-/- $(n=12)$ and controls $(n=10)$. Data are mean $+/-$ SD.

p $<0.05$

** $\mathrm{p} \leq 0.01$

$* * * \mathrm{p} \leq 0.001$ 
Table 3

Tooth Fourier transform-infrared imaging (FTIRI) analysis

\begin{tabular}{cccc}
\hline & Controls (WT) & Hip/Rpl29-/- & $\begin{array}{c}\% \text { null change vs. } \\
\text { control }\end{array}$ \\
\hline A. First Molar & & & \\
Crystallinity & $1.2(+/-0.02)$ & $1.1(+/-0.03)$ & $-\mathbf{8 \%}{ }^{*}$ \\
Mineral/matrix & $9.4(+/-0.97)$ & $10(+/-1.12)$ & $6 \%$ \\
Crosslinking & $2.9(+/-0.23)$ & $3(+/-0.25)$ & $3 \%$ \\
B. Second Molar & & & \\
Crystallinity & $1.1(+/-0.02)$ & $1.1(+/-0.04)$ & No change \\
Mineral/matrix & $9.8(+/-0.65)$ & $10.2(+/-1.22)$ & $4 \%$ \\
Crosslinking & $3.3(+/-0.49)$ & $3.2(+/-0.26)$ & $-3 \%$ \\
\hline
\end{tabular}

Hip/Rpl29 ${ }^{-/-}(\mathrm{n}=5)$ and WT (n=5). Data are mean $+/-$ SD .

p $<0.05$ 